\title{
Enhanced heat transfer characteristics and performance of composite thermoelectric devices
}

\author{
M. K. Chyu, B. V. K. Reddy, M. Barry \& J. Li \\ Department of Mechanical Engineering and Materials Science, \\ University of Pittsburgh, USA
}

\begin{abstract}
Thermoelectric elements made of semiconductor slices layered onto highly conducting inter-connector materials are termed as Composite Thermoelectric Device (CTED). An integrated TED (iTED) is a CTED with flow channels drilled through an inter-connector, it acts as a heat exchanger, directing the heat transfer between the fluid stream and element legs. In this work, thermoelectric performance of such elements in terms of power output $\mathrm{P}_{0}$, heat input $\mathrm{Q}_{\mathrm{h}}$, conversion efficiency $\eta$, electric current and Ohmic and Seebeck voltages for various hot surface temperatures $T_{h}$, load resistances $R_{L}$ and semiconductor slice sizes $\mathrm{d}$ has been investigated using both analytical and numerical methods. The thermoelectric performance of CTED has been compared with a geometrically equivalent conventional TED element. For an optimum load resistance $\mathrm{R}_{\text {optmL }}$, an increase in $\mathrm{T}_{\mathrm{h}}$ showed a substantial increase in $\mathrm{P}_{0}$ and a minor increase in $\mathrm{Q}_{\mathrm{h}}$. At $\mathrm{T}_{\mathrm{h}}=550 \mathrm{~K}$ and $350 \mathrm{~K}$, the optimum $\eta$ for CTED, $11 \%$ and $9 \%$ respectively lesser and nearly four-fold increase in $\mathrm{P}_{0}$ as compared to the conventional TED is observed. The semiconductor slice size $d$ has a significant effect on the CTED performance: an observed increase in both $\mathrm{P}_{0}$ and $\mathrm{Q}_{\mathrm{h}}$ and decrease in $\eta$ is a result of decreasing $\mathrm{d}$. At fixed $\mathrm{d}=5 \mathrm{~mm}$ and hot stream inlet temperature $(450 \mathrm{~K})$ values, the single-stage iTED showed five-times in $\mathrm{P}_{0}$ and two-fold in $\eta$ for a flow rate $\mathrm{Re}=500$ compared to the values at $\mathrm{Re}=100$. These novel TEDs help extract larger amounts of waste heat, reduce thermal stresses and minimize semiconductor material usage as compared to conventional TEDs.

Keywords: thermoelectrics, waste heat recovery, conventional, composite, integrate, analytical solutions, numerical methods, conversion efficiency, power output, voltage, current.
\end{abstract}




\section{Introduction}

The high demand for energy results in tremendous increase in carbon emissions and waste heat released into the atmosphere. It is estimated that approximately two-thirds of the supplied input energy in fossil-fuel power plants, automobiles, steel furnaces, glass mills, industrial heating and cooling systems is rejected as a waste heat to the surroundings. Therefore, there is a need to explore the novel technologies which must be environmentally friendly, small size, stable and reliable. Thermoelectric solid-state technology is one of the viable technologies to extract energy from waste heat and also helps to mitigate carbon foot-print and green-house gas emissions.

Thermoelectric devices are constructed by joining two dissimilar electrically and thermally conductive materials at a junction. Using the Seebeck effect, these devices work as electric power generators when the two junctions are exposed to a temperature differential [1]. Since 1950, the use of semiconductors has resulted in major advances in thermoelectric power generation. With the present thermoelectric materials having figures of merit $\mathrm{ZT} \approx 1.5$, the conversion efficiencies $5-10 \%$ in power generation can be achieved. However, an increase in efficiency for direct heat-to-electricity are sought through the methods of nanostructuring and fabrication [2, 3], novel bulk designs such as cascading [4], multistage [5] and segmented [6-8] TEDs and use of new materials [1].

Using cascading TEDs, Kaibe et al. [4] showed that an efficiency of $12.1 \%$ can be achieved with hot side temperature $\mathrm{T}_{\mathrm{h}}=550^{\circ} \mathrm{C}$ and cold side temperature $\mathrm{T}_{\mathrm{c}}=30^{\circ} \mathrm{C}$. Over a temperature range of 300-973 K, Crane et al. [6] developed a segmented TED and obtained a conversion efficiency of $15 \%$. Moreover, for $\mathrm{T}_{\mathrm{h}}$ $=973 \mathrm{~K}$ and $\mathrm{T}_{\mathrm{c}}=300 \mathrm{~K}$, El-Genk et al. [7] showed a peak conversion efficiencies of $16.69 \%$ and $7.4 \%$ for skutterudite and SiGe segmented thermoelectric generators, respectively. Crane and Lagrandeur [8] also recently investigated the segmented TEDs in automotive waste heat recovery systems and achieved a power output of $125 \mathrm{~W}$ operating at inlet exhaust gas temperature of $650^{\circ} \mathrm{C}$.

Over the past few decades, TEDs have been extensively studied using analytical solutions for simplified cases even though electric and temperature fields are multidimensional in TEDs. Since heat flux and current density are not in parallel, the three-dimensional (3D) thermoelectric phenomenon substantially effects the overall TED performance. In the articles [9-11], it has been shown that $3 \mathrm{D}$ simulations are more accurate in real system design and optimization of TEDs. Harris et al. [9] using finite volume based code investigated the device performance with the presence of inert gas and insulating material considering both thermal and electrical contact resistances at the interfaces. $\mathrm{Hu}$ [10] numerically studied the characteristics of two compact gas-phase heat exchangers placed at hot and cold sides of the TED to enhance a heat transfer and fluid flow while minimizing thermal stresses. Chen et al. [11] recently implemented a 3D numerical model for thermoelectric generators in FLUENTUDS environment taking temperature-dependent thermoelectric properties and non-linear fluid-thermal-electric multi physics coupled effects. 
For the systems such as automobile exhaust gases, power plants and industrial processes where large amounts of waste heat are available, the high conversion efficiency of the TED alone is not sufficient; the device should also be capable of extracting more of the available waste heat. A potential and promising approach is novel composite TED (CTED) design. CTEDs are similar to conventional TEDs in that they use p- and n-type semiconducting materials, but differ in that a highly conducting metal is laminated between the semiconducting materials. The benefit of this design resides in decrease in total internal electrical resistivity; use substantially less rare-earth materials to achieve nearly same performance as conventional TEDs. To further increase the performance of CTED, an integrated TED (iTED) is fabricated by bonding a conducting metal with flow channels between both p-type and n-type thermoelectric materials. This in-flow design capitalizes on reduced elemental resistances, the need for auxiliary ceramic plates, grease and external heat exchangers as in conventional designs and thus increased system reliability. Therefore, in this paper, keeping these salient points in mind, initially the performance of CTEDs using both analytical and numerical methods and then followed by thermoelectric characteristics of iTED applied to waste heat recovery with numerical solutions have been investigated for various load resistances, hot surface temperatures, semiconductor slice sizes and fluid flow configurations.

\section{Analytical solutions for composite thermoelectric device}

Consider a thermoelecric elemental length 'dy' with a constant cross-sectional area $\mathrm{A}(\mathrm{W} \times \mathrm{D})$ as shown in Fig. 1a. This element is experiencing a temperature gradient (-dT/dy) and carrying an electric current I. The element side walls are kept adiabatic. The one-dimensional governing equation for heat transport along an element subjected to assumptions: thermoelectrical properties are treated as constants, the material is homogeneous and isotropic, is written as

$$
\frac{d^{2} T}{d y^{2}}+\frac{\rho I^{2}}{k A^{2}}-\frac{\tau I}{k A} \frac{d T}{d y}=0
$$

In eqn (1), the second and third on the left hand side represent the Joule heating and Thomson effects, respectively.

A composite thermoelectric element consists of two n-types bulk crystalline semiconductors connected in series via conductor as shown in Fig. 1b. The governing equation for heat transport in the inter-connector has been taken from eqn (1) by dropping the Thomson effect term. Using eqn (1) and the associated boundary conditions: top and lower connectors are subjected to temperatures $T_{h}$ and $\mathrm{T}_{\mathrm{c}}$, respectively; at the interface between the semiconductor and conductor materials, the continuity of current density, temperature and heat flux conditions are imposed; the temperature distribution in composite element (Fig. 1b), the interface temperatures and the hot side heat transfer rate evaluated using the analytical solutions are written as:

For the top semiconductor slice: $\quad \theta(Y)=C_{1}+C_{2} e^{D_{1} Y}+M_{1}$ 

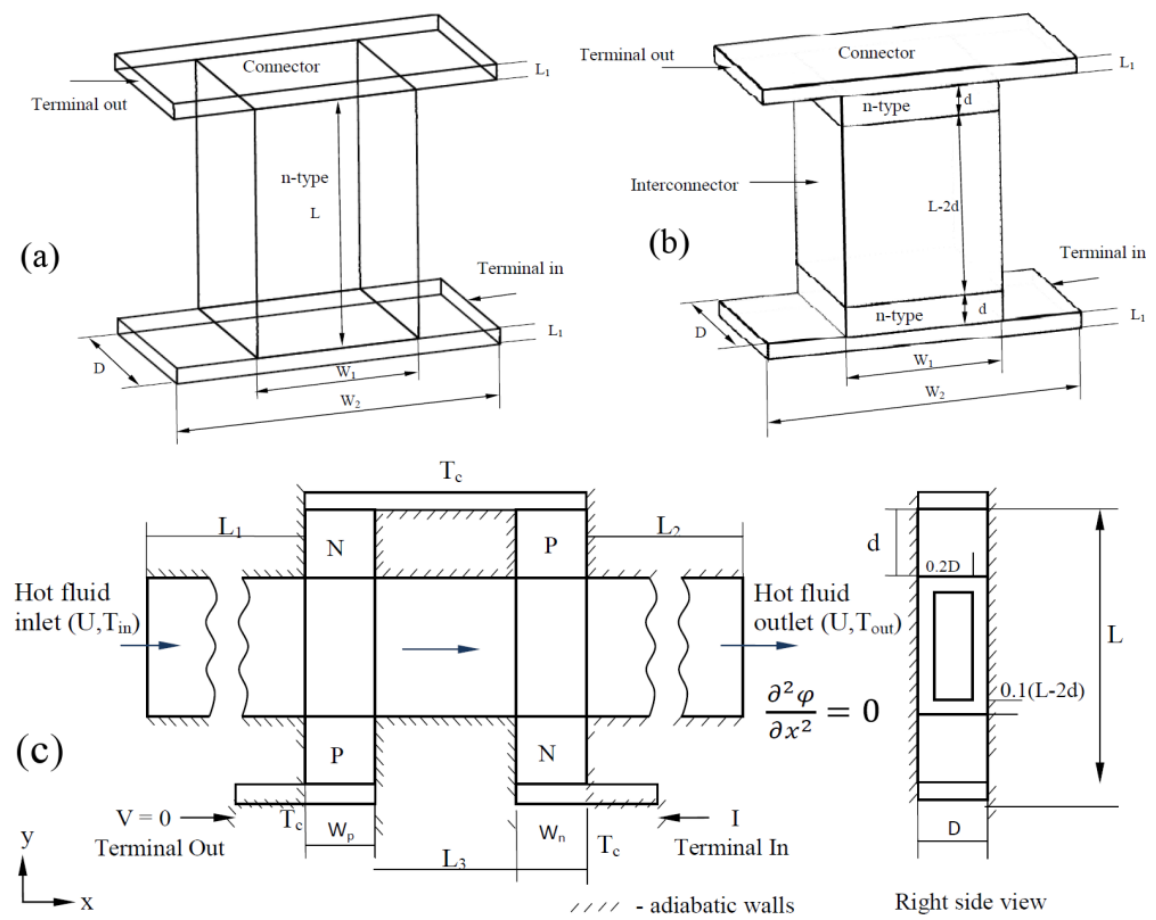

Right side view

Figure 1: $\quad$ Schematics of (a) convenctional (b) composite and (c) integrated TEDs.

where, $Y=\frac{y}{L}, \theta=\frac{T-T_{c}}{T_{h}-T_{c}}, \Gamma=\frac{\tau I L}{k A}, \chi=\frac{I^{2} \rho L^{2}}{k A^{2}\left(T_{h}-T_{c}\right)}, D_{1}=\Gamma_{s 1}, M_{1}=\left(\frac{\chi}{\Gamma}\right)_{s 1}$, $C_{1}=\frac{-e^{D_{1} \delta}-M_{1} \delta+\theta_{1}}{1-e^{D_{1} \delta}}, C_{2}=\frac{1-\theta_{1}+M_{1} \delta}{1-e^{D_{1} \delta}}$ and $\delta=\frac{d}{L}$.

For the inter-connector: $\quad \theta(Y)=C_{1}+C_{2} Y-\frac{\chi_{c} Y^{2}}{2}$

In eqn (3), $C_{1}=\frac{\theta_{1}(1-\delta)-\theta_{2} \delta}{1-2 \delta}-\frac{\chi_{c} \delta(1-\delta)}{2}$ and $C_{2}=\frac{-\theta_{1}+\theta_{2}}{1-2 \delta}-\frac{\chi_{c}}{2}$.

For the bottom semiconductor slice: $\quad \theta(Y)=C_{1}+C_{2} e^{D_{2} Y}+M_{2}$

Here, $D_{2}=\Gamma_{s 2}, M_{2}=\left(\frac{\chi}{\Gamma}\right)_{s 2}, C_{1}=\frac{\theta_{2}-M_{2}\left(1-\delta-e^{-D_{2} \delta}\right)}{1-e^{-D_{2} \delta}}, C_{2}=\frac{-\left(\theta_{2}+M_{2} \delta\right)}{e^{D_{2}}\left(1-e^{-D_{2} \delta}\right)}$

The interface temperatures $\theta_{1}$ and $\theta_{2}$, respectively are

$$
\theta_{1}=\left[1+\frac{k_{s 2}}{k_{c}} \frac{(1-2 \delta)}{\left(e^{D_{2} \delta}-1\right)} D_{2}\right] \theta_{2}-\frac{k_{s 2}}{k_{c}} \frac{(1-2 \delta)}{\left(e^{D_{2} \delta}-1\right)} M_{2}\left[e^{D_{2} \delta}-1-\delta D_{2}\right]
$$


and $\theta_{2}=\frac{A+M_{2}\left[\frac{e^{D_{2} \delta}-1-\delta D_{2}}{e^{D_{2} \delta}}-1 \frac{k_{s 2}}{k_{s 1}}\left[e^{-D_{1} \delta}-1-D_{1} \frac{k_{s 1}}{k_{c}}(1-2 \delta)\right]\right.}{\frac{D_{2}}{\left(e^{D_{2} \delta}-1\right)} \frac{k_{s 2}}{k_{s 1}}\left[e^{-D_{1} \delta}-1-D_{1} \frac{k_{s 1}}{k_{c}}(1-2 \delta)\right]-D_{1}}$

where, $A=\chi_{c}(1-2 \delta)^{2}\left[\frac{k_{c}}{k_{s 1}} \frac{\left(e^{-D_{1} \delta}-1\right)}{1-2 \delta}-\frac{D_{1}}{2}\right]-D_{1}\left[1+M_{1} \delta\right]-M_{1}\left(e^{-D_{1} \delta}-1\right)$.

The heat transfer rate at the hot surface is written as

$$
Q_{h}=\alpha_{s 1} I T_{h}+\frac{k_{s 1} A\left(T_{h}-T_{c}\right)}{L}\left[\frac{\left(-1+\theta_{1}-M_{1} \delta\right) D_{1}}{1-e^{D_{1}}}-M_{1}\right]
$$

The power generation from the composite TED is evaluated as $P_{0}=I^{2} R_{L}$

where, $\mathrm{I}$ is generated electric current $I=\left(-\alpha_{s 1}\left(T_{h}-T_{1}\right)-\alpha_{s 2}\left(T_{2}-T_{c}\right)\right) /\left(R_{i}+R_{L}\right)$.

where, $R_{i}$ is the internal resistance $R_{i}=\left(\rho_{s 1} d+\rho_{c}(L-2 d)+\rho_{s 2} d\right) / A$.

\section{Numerical solutions for thermoelectric devices}

In this section, the fluid-thermo-electric coupled field three-dimensional (3D) numerical methods for thermoelectric devices (TED) considerering Joule heating, Peltier and Thomson effects, along with the temperature dependent thermoelectric property variations, have been implemented.

\subsection{Geometry, governing equations and boundary conditions}

The 3D schematics of composite TED (CTED) and integrated TED (iTED) being investigated are shown in Figs. 1(b) and (c), respectively. The top surface of CTED upper connector is subjected to a hot temperature $\mathrm{T}_{\mathrm{h}}$ while the bottom surface of CTED lower connector and top and bottom surfaces of iTED are set at a cold temperature $T_{c}$. The other surfaces as shown in Fig. 1 are kept adiabatic. In the iTED, a hot fluid with temperature $T_{i n}$ and uniform velocity $U$ enters the inlet and flows through inter-connector channel and leaves at the exit of the domain (Fig. 1c). The inter-connector inner walls and left and right side surfaces are alone responsible for heat transfer from the hot fluid to thermoelectric elements. The end surfaces of connectors act as terminals for load resistance $R_{L}$.

The mass, momentum and heat transport in the fluid flow domain are govern by the $3 \mathrm{D}$ formulation of conservation equations subjected to the following assumptions. The flow is steady, laminar and incompressible. The thermophysical properties of fluid (air) are kept constant. Similarly, the current flow and heat transport in the thermoelectric material are govern by the continuity of current density and the energy equations subjected to certain assumptions. The materials are heterogeneous, isotropic and the thermoelectric properties are specified as polynomial functions of temperature [12]. 
The set of equations governing the fluid flow and heat transport in the fluid domain and the thermo-electric phenomenon in the conductor and semiconductor materials under steady-state conditions are written as:

- Continuity equation:

$$
\text { Fluid region: } \quad \nabla . v=0
$$

Conductor and semiconductor $[1]: \quad \nabla . J=0$

- Momentum equation:

$$
\rho_{f} \cdot(v \nabla v)=\nabla P+\mu \nabla^{2} v
$$

- Energy equation:

$$
\begin{gathered}
\text { Fluid region: } \quad\left(\rho c_{P}\right)_{f}(v \nabla T)=\nabla \cdot\left(k_{f} \nabla T\right) \\
\text { Semiconductor [1]: } \nabla(k \nabla T)+\rho J^{2}-T J \cdot\left[\left(\frac{\partial \alpha}{\partial T}\right) \nabla T+(\nabla \alpha)_{T}\right]=0
\end{gathered}
$$

The second and third terms in eqn (13) represent the Joule heating, Peltier and Thomson effects respectively. In the conductor material, the third term in the left hand side of eqn (13) will be omitted. Using the non-ohmic current-voltage relation, the total electric potential is the summation of electrostatic and Seebeck potentials and is calculated as

$$
\nabla V=\nabla V_{O V}+\nabla V_{S V}=-\rho J-\alpha \nabla T
$$

With respect to the geometry shown in Fig. 1, the associated thermal and electrical boundary conditions are: the 'out' terminal subjected to zero potential while the 'in' terminal kept at constant current density and is evaluated as

$$
J=\frac{I}{A_{\xi}}=\frac{V_{0}}{A_{\xi}\left(R_{i}+R_{L}\right)} \quad \text { and } \quad \frac{\partial T}{\partial \xi}=0
$$

where, $\mathrm{R}_{\mathrm{i}}$ the total internal resistance due to semiconductor slices, connectors and inter-connectors materials and $\mathrm{R}_{\mathrm{L}}$ is the external load resistance. $\mathrm{V}_{0}$ is the total built-in open circuit voltage at no-load condition known as Seebeck voltage. $\xi$ denotes the direction normal to the corresponding surface.

For the up and down streams of the iTED, sufficient buffer lengths $5 \mathrm{~W}$ and $20 \mathrm{~W}$ are provided respectively to achieve no thermal 'back' diffusion at the inlet and outflow condition at the exit of the flow domain. At the interface between the semiconductor and connector or inter-connector materials, the continuity of temperature, current density and the heat flux conditions are imposed.

The TEDs power output $\mathrm{P}_{0}$, heat input $\mathrm{Q}_{\mathrm{h}}$ and efficiency $\eta$ are evaluated as:

$$
P_{0}=I^{2} R_{L}, \quad Q_{h}=\left\{\begin{array}{cc}
-k A_{\xi} \frac{\partial T}{\partial \xi} & C T E D \\
-\sum_{i=1,2} \frac{1}{A s_{i}} \int_{A s_{i}} k_{f} \frac{\partial T}{\partial \xi} d A s_{i} & i T E D
\end{array}, \text { and } \eta=\frac{P_{0}}{Q_{h}}\right.
$$




\subsection{Numerical solution procedure}

The numerical simulations are performed using the finite volume formulation of eqns (9) to (13) and the associated constitutive relation eqn (14) along with associated boundary conditions as shown in Fig. 1 and eqn (15) in ANSYS-FLUENT UDS (User Defined Scalar) environment. The pressure-velocity coupling is handled using SIMPLE algorithm [13]. The spatial descretisation of the convective and diffusion terms with power law scheme and pressure term with standard scheme are done, respectively. The geometric models and mesh are generated in Gambit 2.4. The Seebeck potential and Ohmic electric potential distributions (eqn (14)), continuity of current density (eqn (10)) and the Peltier term (eqn (13)) are calculated using UDS fields. The Ohmic heating, Peltier and Thomson effects are modeled as source terms (eqn (13)). The electric current is evaluated based on the open-circuit Seebeck voltage produced at the given load resistance $\mathrm{R}_{\mathrm{L}}$ (eqn (15)). The further description on numerical implementation of the TED in the FLUENT has been given in [11].

The convergence criteria for mass, momentum, energy, current density, Seebeck and Ohmic electric potentials are set as $10^{-5}, 10^{-5}, 10^{-15}, 10^{-10}, 10^{-10}$ and $10^{-10}$, respectively. Suitable grid sizes were chosen for further numerical simulations on both TEDs after performing grid independence study. Grid sizes with 220000 and 1574424 cells are used for CTED and iTED respectively to generate further results. The orthogonal and nonuniform grids are used. The 3D thermoelectric model has been validated with published results of a thermoelectric generator for the cases of (a) constant properties [12] and (b) temperature-dependent properties [14] and are given in Table 1.

Table 1: Comparison of thermoelectric generator parameters at $\mathrm{T}_{\mathrm{h}}=427{ }^{\circ} \mathrm{C}$ and $\mathrm{T}_{\mathrm{c}}=27^{\circ} \mathrm{C}$.

\begin{tabular}{|c|c|c|c|c|c|}
\hline Quantity & $\begin{array}{c}\text { Analytical } \\
{[12]}\end{array}$ & $\begin{array}{l}\text { ANSYS } \\
\text { (a) [14] }\end{array}$ & $\begin{array}{l}\text { ANSYS } \\
\text { (b) [14] }\end{array}$ & $\begin{array}{l}\text { Present } \\
\text { (a) }\end{array}$ & $\begin{array}{l}\text { Present } \\
\text { (b) }\end{array}$ \\
\hline $\mathrm{Q}_{\mathrm{h}}, \mathrm{W}$ & 13.04 & 13.03 & 11.07 & 13.0 & 10.72 \\
\hline $\mathrm{P}_{0}, \mathrm{~W}$ & 1.44 & 1.43 & 1.05 & 1.43 & 1.07 \\
\hline$\eta\left(\mathrm{P}_{0} / \mathrm{Q}_{\mathrm{h}}\right), \%$ & 11 & 11 & 9.5 & 11 & 9.98 \\
\hline $\mathrm{I}, \mathrm{A}$ & 19.2 & 19.1 & 16.4 & 19.09 & 16.5 \\
\hline
\end{tabular}

\section{Results and discussion}

In this section, the thermoelectric performance of conventional, composite and integrated thermoelectric devices (TEDs) using both analytical and numerical solutions have been investigated. For the analytical results the thermoelectric properties are evaluated at averaged temperature of hot and cold surfaces, where as in the case of numerical solutions the properties are varied with temperature. The bulk crystalline n-type (75\% $\left.\mathrm{Bi}_{2} \mathrm{Te}_{3} 25 \% \mathrm{Bi}_{2} \mathrm{Se}_{3}\right)$ and p-type $\left(25 \% \mathrm{Bi}_{2} \mathrm{Te}_{3}\right.$ $75 \% \mathrm{Sb}_{2} \mathrm{Te}_{3}, 1.75 \%$ excess $\mathrm{Se}$ ) semiconductors and copper as a connector and inter-connector materials are used to analyze various TEDs performance. 


\subsection{Composite Thermoelectric Device (CTED)}

As shown in Fig. 1(b), the connector size $\left(\mathrm{W}_{2}=10 \mathrm{~mm}, \mathrm{D}=5 \mathrm{~mm}\right.$ and $\mathrm{L}_{1}=$ $0.5 \mathrm{~mm})$, the distance between the connectors $(\mathrm{L}=8 \mathrm{~mm})$, the cross-sectional area of the element $\left(5 \times 5 \mathrm{~mm}^{2}\right)$ and the cold wall temperature $\left(\mathrm{T}_{\mathrm{c}}=300 \mathrm{~K}\right)$ are fixed for further CTED simulated results. The influence of hot wall temperature $350 \leq \mathrm{T}_{\mathrm{h}}(\mathrm{K}) \leq 550$, the load circuitry resistance $10^{-4} \leq \mathrm{R}_{\mathrm{L}}(\mathrm{Ohms}) \leq 10^{-1}$ and the size of n-type material $0.25 \leq \mathrm{d}(\mathrm{mm}) \leq 3$ on the thermoelectric performance of CTED has been analysed and compared with geometrically equivalent convenctional TED in terms of the power output $\mathrm{P}_{0}$, heat input $\mathrm{Q}_{\mathrm{h}}$, conversion efficiency $\eta$, produced electrical current I and the Ohmic and Seebeck voltages V.

For fixed $d=1 \mathrm{~mm}$, Figs. 2(a)-(e) show the variation of $\mathrm{P}_{0}, \mathrm{Q}_{\mathrm{h}}, \eta, \mathrm{I}$ and Ohmic $V$ with $R_{L}$ for two hot wall temperatures $T_{h}$. In these Figures, the solid lines with filled circles and empty circles represent the numerical and analytical results of CTED respectively, while the solid lines with filled squares describes convectional TED numerical results. In Fig. $2 \mathrm{a}$ and c, it is shown that there exists a maximum power output $\mathrm{P}_{0}$ at $\mathrm{R}_{\mathrm{L}}=\mathrm{R}_{\mathrm{i}}$ and optimum $\eta$ at a particular resistance $R_{L}$ known as optimum load resistance $R_{o p t m L}$ for a given hot wall temperature $T_{h}$, respectively. The $R_{\text {optmL }}$ increases with an increase in $T_{h}$ values while all other parameters are kept constant. The same behaviour is observed in both the CTED and conventional TEDs. However, more $\mathrm{P}_{0}$ and less $\mathrm{R}_{\text {optmL }}$ values are predicted when compared to conventional TEDs. It is also observed that the optimum $\eta$ value of CTEDs are $11 \%$ and $9 \%$ lesser than conventional TEDs at $\mathrm{T}_{\mathrm{h}} 550 \mathrm{~K}$ and $350 \mathrm{~K}$, respectively. This is due to the mutual interplay of $\mathrm{P}_{0}$ and $\mathrm{Q}_{\mathrm{h}}$. At a given $\mathrm{T}_{\mathrm{h}}$ value, $\mathrm{Q}_{\mathrm{h}}$ decreases with $\mathrm{R}_{\mathrm{L}}$ and also $\mathrm{Q}_{\mathrm{h}}$ decreases with decreasing $\mathrm{T}_{\mathrm{h}}$ values. The CTED exhibited nearly four times the value of $\mathrm{Q}_{h}$ as compared to the conventional TED as shown Fig. 2b. The numerical results are showed minor difference from analytical predictions and it is due to inclusion of temperature dependent properties variation and end connectors in the numerical analysis.

From Fig. 2d and e, it is observed that for a given $R_{L}$ value, an increase in $T_{h}$ results in an increase in both $I$ and $V$ values. However, at a given $T_{h}$ with an increase in $R_{L}$ leads to a decrease in both $\mathrm{I}$ and $\mathrm{V}$ values and eventually approaches zero values at larger $\mathrm{R}_{\mathrm{L}}$ value. The same behaviour is also observed in the conventional TED. Moreover, the I value in the CTED is higher when compared to the conventional TED and the opposite response is observed in $\mathrm{V}$. This is due to the total internal resistance $\left(\mathrm{R}_{\mathrm{i}}=\mathrm{R}_{\mathrm{c}}+\mathrm{R}_{\mathrm{n}}\right)$ of CTED being less compared to the conventional TED. It is also observed that the difference between the ordinate values of the CTED and the conventional TED decreases with $R_{L}$ in case of $I$, whereas the same trend has not been shown for $V$. The reason is the interplay between the $R_{i}$ and $R_{L}$ values.

The influence of semiconductor slice size $d$ at $R_{o t m L}$ values on thermoelectric performance of TEDs is shown in Fig. 3. The $\mathrm{P}_{0}, \mathrm{Q}_{\mathrm{h}}$ and I show exponential decay with an increase in $\mathrm{d}$. Conversely, $\eta$ shows an exponential increase trend. While the temperature differential $T_{h}-T_{c}$ and element height $L$ are maintained invariant the increment in $\mathrm{d}$ results the performance parameters values of the CTED approach the conventional TED values. For brevity, under the similar 
conditions, the conventional TED design performance values are mentioned in the respective figures. It is observed that $d$ has a significant effect on the thermoelectric performance of the CTED when $\mathrm{d}<1 \mathrm{~mm}$. However, when $>3 \mathrm{~mm}$, it shows minimal change in $\mathrm{P}_{0}, \mathrm{Q}_{\mathrm{h}}, \eta$ and $\mathrm{I}$ predictions. This is due to the substantial change in the CTED total $\mathrm{R}_{\mathrm{i}}$ value.
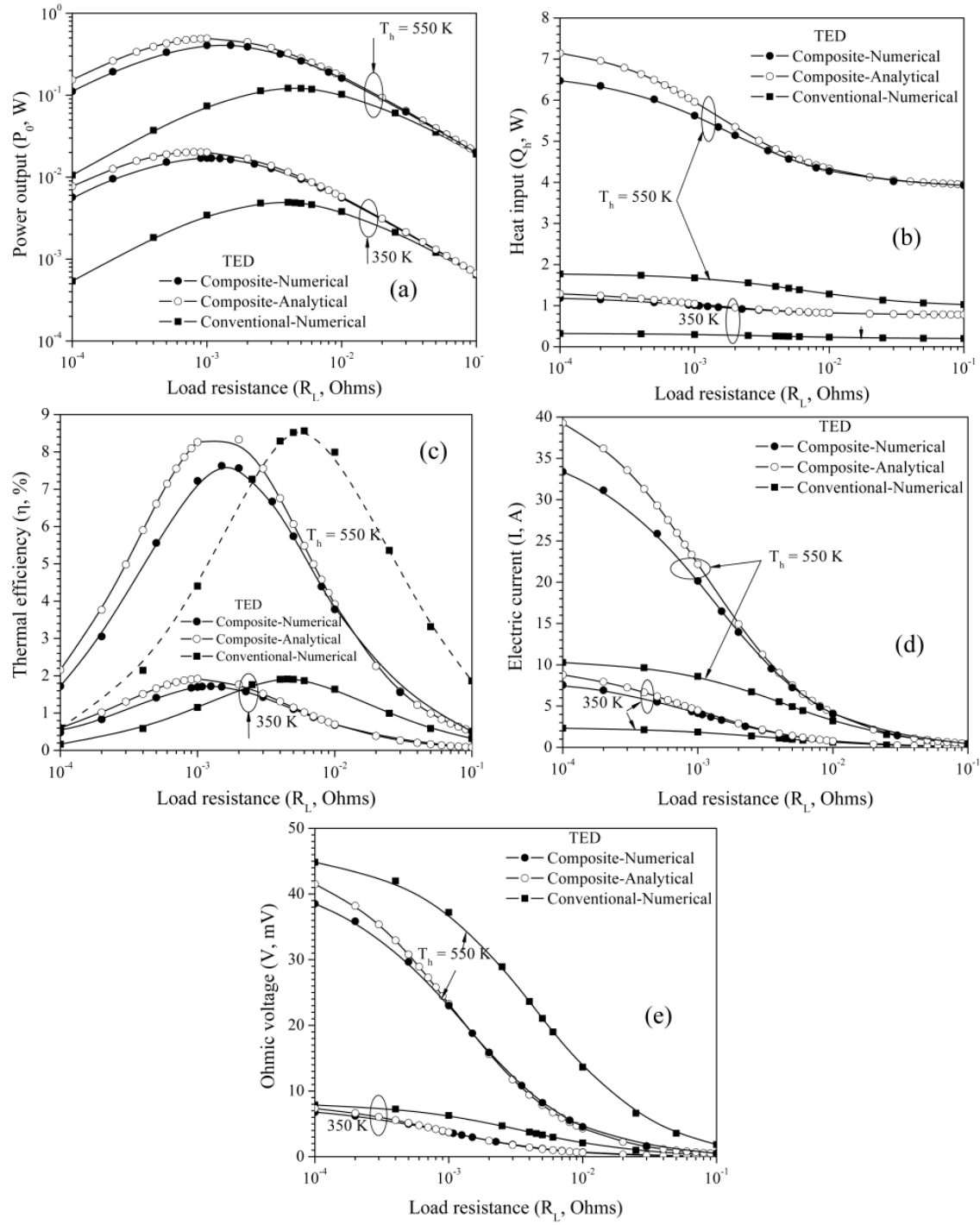

Figure 2: $\quad$ Effect of load resistance $\mathrm{R}_{\mathrm{L}}$ on (a) power output (b) heat input (c) efficiency (d) electrical current and (e) voltage for various $T_{h}$ values $[\mathrm{d}=1 \mathrm{~mm}]$. 

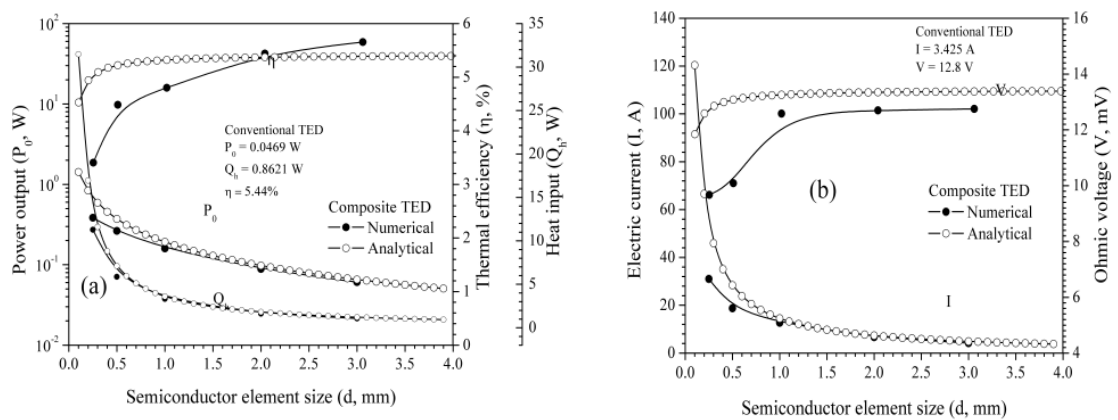

Figure 3: The variation of (a) heat input, power output and efficiency and (b) electric current and voltage for different semiconductor sizes $\mathrm{d}\left[\mathrm{T}_{\mathrm{h}}=450 \mathrm{~K}\right]$.

\subsection{Integrated Thermoelectric Device (iTED)}

The iTED parameters: hot fluid inlet temperature $T_{\text {in }}=550 \mathrm{~K}$; semiconductor ( $\mathrm{n}$ type and p-type) element size $\mathrm{d}=5 \mathrm{~mm}$; the height of module $\mathrm{H}=20 \mathrm{~mm}$; connector thickness $\mathrm{t}=1.5 \mathrm{~mm}$; the semiconductor and inter-connector depth $\mathrm{D}$ $=5 \mathrm{~mm}$ and width $\mathrm{W}=5 \mathrm{~mm}$; the distance between legs $\mathrm{L} 3=10 \mathrm{~mm}$; the bottom connectors length $10 \mathrm{~mm}$ and the top connector length $20 \mathrm{~mm}$ are kept constant.
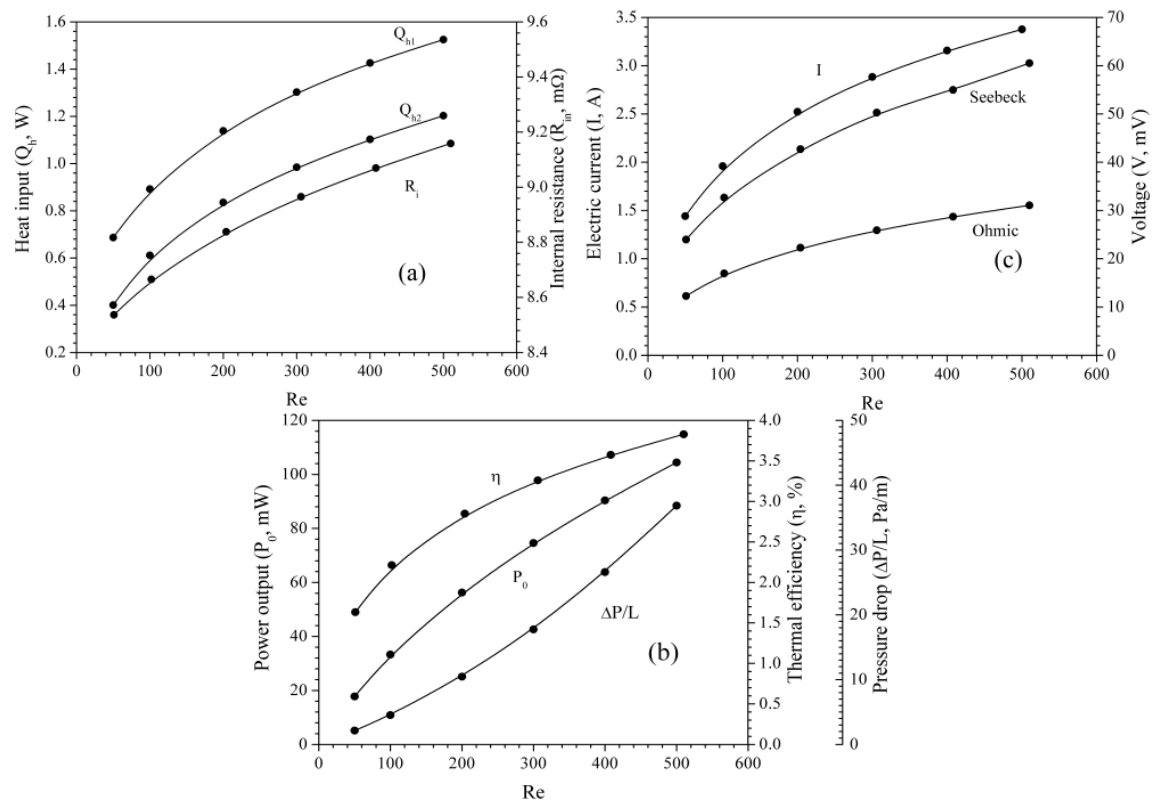

Figure 4: $\quad$ Effect of Re on (a) heat input and load resistance (b) power output and efficiency and (c) electric current and voltage $\left[\mathrm{T}_{\mathrm{in}}=550 \mathrm{~K}\right.$, $\left.\mathrm{d}=5 \mathrm{~mm}, \mathrm{R}_{\mathrm{L}}=\mathrm{R}_{\mathrm{i}}\right]$. 
The effects of several flow rates $50 \leq \operatorname{Re}\left(\rho \mathrm{UD}_{\mathrm{h}} / \mu\right) \leq 500$ on iTED performance in terms of thermoelectric characteristics $\left(\mathrm{P}_{0}, \mathrm{Q}_{\mathrm{h}}, \eta, \mathrm{I}\right.$ and $\left.\mathrm{V}\right)$ have been shown in Fig. 4(a)-(c). The change in total internal resistance $\mathrm{R}_{\mathrm{i}}$ and the pressure drop $\Delta \mathrm{P} / \mathrm{L}$ with Re is also depicted in fig. 4(a) and (b), respectively. Here, in fig. 4(a), $\mathrm{Q}_{\mathrm{h} 1}$ and $\mathrm{Q}_{\mathrm{h} 2}$ represent the total heat transferred to the left and right legs of the iTED module, respectively. For a given $\mathrm{T}_{\text {in }}=550 \mathrm{~K}$, an increase in Re shows an enhancement in $Q_{h}$ and hence, resulted higher $T_{h}-T_{c}$ values. This increase in $T_{h}-$ $\mathrm{T}_{\mathrm{c}}$ value helps to achieve larger built-in Seebeck voltage and which in turn results higher power output $\mathrm{P}_{0}$. At $\mathrm{Re}=500$, five-times in $\mathrm{P}_{0}$ and two-fold in $\eta$ are obtained compared to the values at. $R e=100$ The electrical characteristics I and $\mathrm{V}$ have shown similar behaviour with Re. It is also noticed that at given Re, the Ohmic voltage drop is higher than the Seebeck voltage drop value. Further, the flow rate in the specified range has minimal effect on $R_{i}$ predictions and it is shown nearly $7 \%$ variation. This change alone is due to the variation in electrical resistivity value with temperature. The $\Delta \mathrm{P} / \mathrm{L}$ shows a non-linear behaviour with $\mathrm{Re}$ and it is due to the inclusion of additional pressure drop for the recirculation zones formation in the main flow channel.

\section{Conclusions}

Using analytical and numerical solutions, the performance of conventional, composite and integrated thermoelectric devices (TEDs) considering Joule heating, Peltier and Thomson effects, along with the temperature dependent thermoelectric property variations, have been invetsigated. The influences of hot surface temperature $T_{h}$, the semiconductor slice size $d$ and the hot fluid flow rate Re on the thermo-hydraulic-electrical characteristics i.e. power output $\mathrm{P}_{0}$, heat input $\mathrm{Q}_{\mathrm{h}}$, conversion efficiency $\eta$, pressure drop $\Delta \mathrm{P} / \mathrm{L}$, produced electric current I, Ohmic and Seebeck voltages $\mathrm{V}$ of various TED designs have been studied.

The difference in $\mathrm{P}_{0}$ 's of CTED and conventional TED is decreased with an increase in load resistance $R_{L}$. It is showed that $P_{0}$ reaches a maximum value when the internal resistance $R_{i}$ equals $R_{L}$. For the CTED, the $R_{\text {optmL }}$ is lower than conventional TED value. For both TEDs, an increment in the $T_{h}$ showed a marginal increment in $R_{\text {optmL. }}$ It is also observed that for the CTED with $d=1$ $\mathrm{mm}$ case is $11 \%$ and $9 \%$ lower in maximum $\eta$ and nearly four times higher in $\mathrm{Q}_{\mathrm{h}}$ compared to conventional TED values at $\mathrm{T}_{\mathrm{h}} 550 \mathrm{~K}$ and $350 \mathrm{~K}$, respectively.

The $\mathrm{P}_{0}, \mathrm{Q}_{\mathrm{h}}$ and $\mathrm{I}$ values showed an exponential decay response with an increase in $d$, however, an exponential raise in $\eta$ with $d$ is observed. In the limit, $\mathrm{d} \approx$ half of the conventional TED element size, the performance of the CTED reaches conventional TED values. When $\mathrm{d}<1 \mathrm{~mm}$, it is seen that the size of slice has significant effect on the performance of the CTED design.

For the iTED configuration, as Re increases, there is an enhancement in $Q_{h}$. This higher $\mathrm{Q}_{\mathrm{h}}$ helps to achieve a larger Seebeck voltage through temperature differential $T_{h}-T_{c}$ and which in turn produces higher $P_{0}$ value. At a given $R e$, the Ohmic voltage drop is higher than the Seebeck voltage drop value. The $\Delta \mathrm{P} / \mathrm{L}$ shows a non-linear behaviour with $\mathrm{Re}$ and it is due to an additional pressure drop occurance for the recirculation zones formed in the main channel flow. 
The novel CTED and iTEDs help extract larger amounts of waste heat, reduce thermal stresses and minimize rare-earth material usage as compared to conventional TEDs, without compromising their efficiencies and performance.

\section{References}

[1] Rowe, D.M., (ed.) Thermoelectrics Handbook Macro to Nano. CRC Press, Taylor \& Francis Group, Boca Raton, 2006.

[2] Snyder, G.J. and S.Toberer, E., Complex thermoelectric materials. Nature Materials, 7, pp. 105-114, 2008.

[3] Tritt, T.M., Thermoelectric phenomena, materials, and applications. The Annual Review of Materials Research, 41, pp. 433-448, 2011.

[4] Kaibe, H., Aoyama, I., Mukoujima, M., Kanda, T., Fujimoto, S., Kurosawa, T., Ishimabushi, H., Ishida, K., Rauscher, L., Hata, Y. and Seijirou, Development of thermoelectric generating stacked modules aiming for $15 \%$ of conversion efficiency. Int. Conference on Thermoelectrics, IEEE, pp. 227-232, 2005.

[5] Liang, G., Zhou, J. and Huang, X., Analytical model of parallel thermoelectric generator. Applied Energy, 88, pp. 5193-5199, 2011.

[6] Crane, D.T., Kossakovski, D. and Bell, L.E., Modeling the building blocks of a $10 \%$ e_cient segmented thermoelectric power generator. Journal of Electronic Materails, 38(7), pp. 1382-1386, 2009.

[7] El-Genk, M.S., Saber, H.H. and Caillat, T., Efficient segmented thermoelectric unicouples for space power applications, Energy Conversion and Management, pp. 1755-1772, 2003.

[8] Crane, D.T. and Lagrandeur, J., Progress Report on BSST-Led US Department of Energy Automotive Waste Heat Recovery Program, Journal of Electronic Materials, 39 (9), pp. 2142-2148, 2010.

[9] Harris, R., Hogan, T., Schock, H.J. and Shih, T., Heat transfer and electric current flow in a thermoelectric couple. 44th Aerospace Sciences Meeting and Exhibition, AIAA: Reno, Nevada, 575, 2006.

[10] Hu, K.S.Y., Heat transfer enhancement in thermoelectric power generation. M.s, Iowa State University, 2009.

[11] Chen, M., Rosendahl, L.A. and Condra, T., A three-dimensional numerical model of thermoelectric generators in fluid power systems. Int. J. Heat and Mass Transfer, 54, pp. 345-355, 2011.

[12] Angrist, S.W., Direct Energy Conversion. Allyn and Bacon Inc. (Boston), 4th edition, 1982.

[13] Patankar, S., Numerical Heat Transfer and Fluid Flow. Taylor and Francis, 1980 .

[14] Antonova, E.E. and Looman, D.C., Finite elements for thermoelectric device analysis in ansys. IEEE Int. Conference on Thermoelectrics, pp. 200-203, 2005. 